\title{
Data Model for Initiatives to Monitor Exposure to Antimicrobials (DataMIME)
}

\author{
Megan T. Patel ${ }^{\star 1}$, Carlos Santos ${ }^{2}$, Ron Price ${ }^{3}$, George Nelson ${ }^{4}$, Lauren Hall ${ }^{5}$, \\ Richard Platt ${ }^{6}$ and William Trick ${ }^{7}$
}

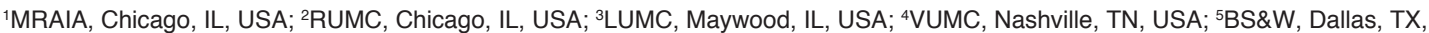
USA; ${ }^{6}$ Harvard Pilgrim, Boston, IL, USA; ${ }^{7} \mathrm{CCHHS}$, Chicago, IL, USA

\section{Objective}

Plan, develop, and pilot an open source system that could be integrated into the PCORnet (PCORI) and Sentinel (FDA) national common data models (CDMs) to generate antimicrobial use (AU) reports submittable to CDC's National Healthcare Safety Network (NHSN). The system included ancillary tables, and data quality and report generation queries. The DataMIME system will allow hospitals to generate comparable AU reports for hospital inpatients.

\section{Introduction}

Despite decades of attempts to promote judicious AU, the US has high rates of per-person antimicrobial consumption, and extremely high rates of carbapenem use ${ }^{1}$. Such profligate use is a key factor in the high rate of antimicrobial-resistant infections seen in US healthcare facilities ${ }^{2}$. Antimicrobial stewardship (AS) programs have been identified as a critical component of intervention strategies ${ }^{3}$. A core component of AS programs is tracking AU, which is needed to monitor trends in use, focus interventions on aberrant behaviors, promote judicious use, and evaluate the effectiveness of interventions. A system designed to extend two national data models would provide a scalable platform for rapid adoption of AU reporting.

\section{Methods}

Virtual meetings were held with all participating sites (five hospitals in IL, LA, and TN) to develop the ancillary tables to capture intrahospital patient movement, and administration of antimicrobial agents. Ancillary tables were designed \& sites populated the tables with calendar year 2016 data. Data characterization was performed to assess overall table statistics, and verify mappings of facility unit locations to NHSN location codes, medications to RxNorm, and routes of administration to one of four SNOMED categories. Additional characterization focused on CDC's NHSN Validation Protocol for the AU module. Analytical queries were developed to produce the output metrics required for submission to the NHSN AU module.

\section{Results}

Two ancillary tables and two look-up tables were developed: a bed information table (Table 1) to capture local location codes with a datetime stamp for precise tracking of patient location; a separate location look-up table allows mapping to other terminologies (Table 2); and, an inpatient drug administration table (Table 3) to capture data from the electronic medication administration record (eMAR) or bar coding medication administration (BCMA) system, utilizing a route of administration look-up table (Table 4). The data model was structured to accommodate use cases with alternative mapping terminologies for local location code, local term for route of administration, local codes for medication, and the option of including the NDC code. All sites populated the ancillary tables. For the bed information table, all sites utilized their ADT table information for patient movement. For the medication administration table, most sites limited the inclusion criteria to the 89 antimicrobial agents required for reporting to the NHSN AU module. Aggregate results from participating sites for facility-wide measures and select antimicrobial agents are presented in Table 5 .

\section{Conclusions}

The data model developed was able to produce the metrics required for reporting to CDC's NHSN AU module. The data dictionary language, implementation guidance, mappings, and queries will be distributed as a tool-kit for other PCORnet and Sentinel sites for reporting to the AU module. In addition, this CDM could allow for the development of additional metrics including excessive use of antibiotic combinations of redundant spectra, syndrome specific antibiotic use, or increased use of excessively broad spectrum antibiotic classes.

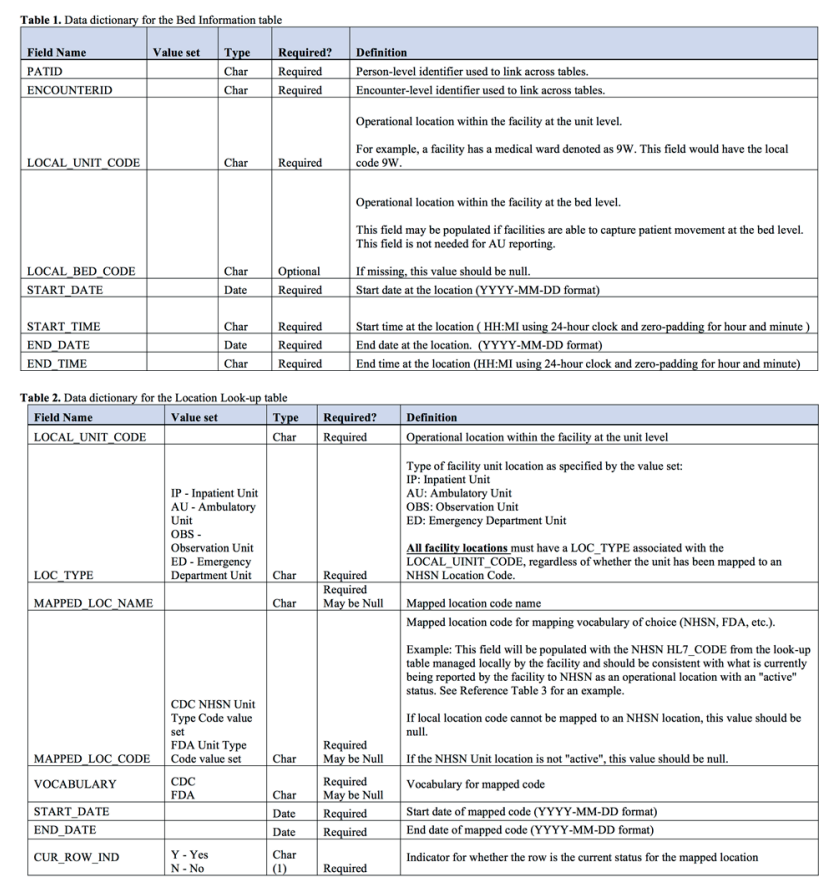




\section{ISDS 2018 Conference Abstracts}

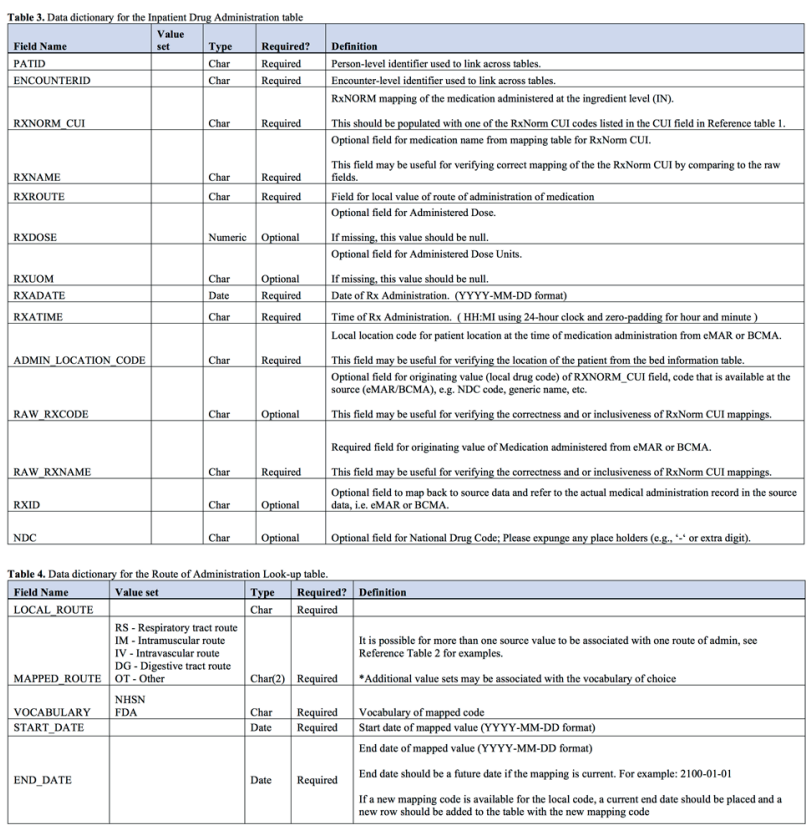

Table 5. Aggregate facility-wide measures for one month's worth of antimicrobial use data.

\begin{tabular}{lcc}
\hline Antimicrobial Agent & $\begin{array}{c}\text { Patient Days } \\
\text { Present }\end{array}$ & $\begin{array}{c}\text { Therapy } \\
\text { Days }\end{array}$ \\
\hline VANC - Vancomycin & 30472 & 1958 \\
PIPERWT - Piperacillin with Tazobactam & 30472 & 1895 \\
CEFAZ - Cefazolin & 30472 & 1305 \\
LEVO - Levofloxacin & 30472 & 1006 \\
METRO - Metronidazole & 30472 & 968 \\
\hline
\end{tabular}

\section{Keywords}

Data Model; Antimicrobial Use; NHSN; PCORnet; Sentinel

\section{Acknowledgments}

Helen Zhang, Alex Patino, Sue Zelisko, Ekta Kishen, Taoran Qiu, Julie Lange, Shazia Sathar, Laura Shockro

\section{References}

1. Van Boeckel TP, Brower C, Gilbert M, Grenfell BT, Levin SA, Robinson TP, Teillant A, Laxminarayan R. Global trends in antimicrobial use in food animals. Proc Natl Acad Sci USA 2015; 112(18):5649-54.

2. CDC. Antibiotic resistance threats in the United States, 2013. Atlanta, GA: US Dept. of HHS, CDC; 2013.

3. Dellit TH, Owens RC, McGowan JE, Gerding DN, Weinstein RA, Burke JP, et al. IDSA and the SHEA Guidelines for Developing an Institutional Program to Enhance Antimicrobial Stewardship. Clin Infect Dis 2007; 44(2):159-77.

\section{*Megan T. Patel}

E-mail:mtoth2@uic.edu 